\title{
Could dentists be the first to diagnose COVID-19 due to oral manifestations?
}

\section{Yasmine Coll ${ }^{* 1}$ and Fatima Elmahgoub ${ }^{2}$}

\section{A commentary on}

\section{Eghbali Zarch R, Hosseinzadeh P.}

COVID-19 from the perspective of dentists: A case report and brief review of more than 170 cases. Dermatol Ther 2021; DOI: 10.1111/ dth.14717.

\begin{abstract}
Data sources A search of electronic databases (PubMed and Google Scholar) was carried out, with publication being set from 2019 to October 2020.

Study selection Titles and abstracts from the original search were reviewed by two reviewers independently. Overall, 17 studies were included in the final analysis.
\end{abstract}

Data extraction and synthesis Data extraction was conducted independently by two reviewers. Studies that were chosen were examined and the following data parameters were included: age and gender, COVID-19 polymerase chain reaction (PCR) test, COVID19 manifestations, treatment for COVID-19, cutaneous lesions, oral manifestations, days after COVID-19 diagnosis, treat/healing or oral symptoms.

Results Seventeen studies were included; 14 articles were case reports, two case series and one systematic review. The results showed that dry mouth, dysgeusia, oral ulcerations and opportunistic infections were among the most common oral manifestations expressed in COVID-19-positive patients.

Conclusion The authors recommended carrying out careful clinical intraoral examinations on both COVID-19-positive patients or any patients requiring dental care, as oral symptoms can still be the only or initial symptom of COVID-19.

\section{Commentary}

The World Health Organisation declared COVID-19 a pandemic on 11 March 2020. Since then, this disease has caused a significant number of deaths in many countries worldwide. The disease is caused by one of the coronaviruses (SARS-CoV-2) which is a contagious respiratory virus. The initial symptoms to be aware of include fever, a new continuous cough and a loss or change in the sense of smell or taste as highlighted by the NHS inform website. ${ }^{1}$

This systematic review suggests that there are a range of oral manifestations that can occur as a result of COVID-19, ranging from dry mouth to opportunistic infections such as Candida albicans. It was highlighted that in some cases, oral symptoms may be the initial or only sign of the disease, and as healthcare
Practice point

- Further research is required into COVID-19 and potential oral manifestations, including onset, duration, and whether or not treatment and intervention is required. professionals, a careful clinical oral examination should be carried out with this in mind.

There are a few issues in the systematic review that could have been avoided to improve its quality: 1) the search was limited to two databases; 2 ) it was not clear whether the risk of bias assessment was addressed, as it did not allude to how disagreements were addressed; and 3) due to the presence of some missing data across all of the studies included, it is difficult to quantitatively analyse and compare oral manifestations and disease patterns (as acknowledged by the authors).

Furthermore, papers were retrieved from an electronic database search only, while grey literature was not searched or included. Only English language studies were included, meaning studies in different languages which could have some potential relevance may have been missed, particularly considering that this is a worldwide pandemic.

When considering risk of bias, it was not disclosed whether or not a tool was used in order to assess the quality or accuracy of the studies included. With COVID-19 being a new condition, the number of studies concerned with oral manifestations are limited and more research is required in this field. The final conclusion is too vague to suggest that we should implement changes to our practice. The outbreak of COVID-19 has caused delays in carrying out oral examinations and oral manifestations may be misdiagnosed or there may be coincidental findings; therefore, more research is required into this topic.

\section{Author affiliations \\ ${ }^{1}$ DCT2, Oral and Maxillofacial Surgery, Crosshouse Hospital, Kilmarnock, UK; ${ }^{2}$ DCT3, Oral and Maxillofacial Surgery, Crosshouse Hospital, Kilmarnock, UK. ${ }^{*}$ Correspondence to: Yasmine Coll}

\section{Reference}

1. World Health Organisation. Technical guidance. 2021. Available online at https:// www.who.int/emergencies/diseases/novel-coronavirus-2019/technical-guidance (accessed March 2021).

Evidence-Based Dentistry (2021) 22, 49.

https://doi.org/ 10.1038/s41432-021-0169-z 